\title{
Modelación del volumen de rodal para especies secundarias en bosques nativos de Mimosa scabrella en la región metropolitana de Curitiba, Paraná, Brasil
}

\author{
Modeling of the stand volume for secondary species in native bracatinga forests \\ in the metropolitan region of Curitiba, Paraná, Brazil
}

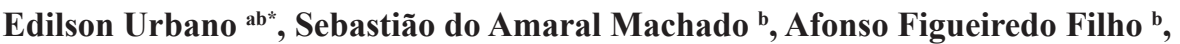 \\ Carlos Roberto Sanquetta ${ }^{b}$, Jorge Danilo Zea-Camaño ${ }^{b}$
}

${ }^{a}$ Universidade Estadual do Mato Grosso do Sul, Unidade Universitária de Aquidauana, Curso de Engenharia Florestal, Rodovia Aquidauana/Uems Km 12, Aquidauana, Mato Grosso do Sul, Brasil, tel.: +55 6739042062.

*Autor de correspondencia: ${ }^{\text {b }}$ Universidade Federal do Paraná, Campus Jardim Botânico, CIFLOMA, Departamento de Ciências Florestais, Rua Lothário Meissner 900,CEP:80210-170, Curitiba, Paraná, Brasil, edurbano2@gmail.com

\section{SUMMARY}

Forest formations known as bracatingais - bracatinga forest - were initially composed only by the Mimosa scabrella - bracatinga which, over the years, has been being replaced by several secondary species. The wood produced in these bracatingais is mainly sold for firewood and charcoal, being an important source of income for the small owners of this region. To facilitate the quantification of volumetric wood production, the objective of this work was to model the volume of firewood with bark per unit of area for the other hardwood species of the bracatingais. The data base comes from 320 plots measured in stands with ages varying from 3 to 20 years, measured between 1996 and 2011 in several municipalities of the metropolitan region of Curitiba. In these plots were measured in all the trees, with at least $5 \mathrm{~cm}$ in diameter, the variables total height and diameter at breast height. The individual volume of each tree in each plot was estimated using equations adjusted for similar forest formations. Twenty nine forest models selected from the forest literature, plus one arithmetic and one logarithmic, were adjusted by the forward stepwise process. The logarithmic equation adjusted by the forward stepwise process was the one with the best overall performance, with coefficient of determination adjusted of 0.992 and standard error of $10.17 \%$, which has no correlation among the residues by the Durbin Watson test and which was validated and considered suitable for estimating the volume per hectare of the hardwood species of bracatingais.

Key words: Mimosa scabrella, volume per hectare, volumetric equations.

\section{RESUMEN}

Los bosques de bracatinga o bracatingais, son formaciones forestales que en su inicio se componen exclusivamente por bracatinga (Mimosa scabrella) que a lo largo de los años va siendo reemplazada por otras especies arbóreas secundarias. La madera producida en estos bracatingais es comercializada principalmente para leña y carbón vegetal y es una fuente importante de renta para los pequeños agricultores de la región metropolitana de Curitiba. Con el propósito de facilitar la cuantificación de leña en estos ambientes, el objetivo de este estudio fue ajustar ecuaciones de volumen con corteza en nivel de rodal para especies secundarias allí presentes. La base de datos procede de 320 parcelas temporales en bracatingais con edades comprendidas entre 3 y 20 años, medidas en el periodo de 1996 a 2011 en varios municipios. Para todos los árboles con diámetro mínimo de $5 \mathrm{~cm}$, fueron medidas las variables altura total y diámetro a la altura del pecho. En total fueron ajustados 29 modelos matemáticos provenientes de la literatura forestal, además de uno aritmético y otro logarítmico por medio del proceso de selección de variables hacia adelante paso a paso "forward stepwise". La ecuación logarítmica ajustada por medio del procedimiento "forward stepwise" fue la de mejor desempeño general, con coeficiente de determinación ajustado de 0,992 y error estándar de 10,17 la cual no tiene correlación entre los residuos por la prueba de Durbin Watson y fue validada estadísticamente para estimar el volumen por hectárea de especies secundarias en rodales de bracatingais.

Palabras clave: Mimosa scabrella, volumen con corteza, ecuaciones volumétricas, forward stepwise.

\section{INTRODUCCIÓN}

Los bracatingais son formaciones forestales compuestas en la fase inicial predominantemente por la especie $\mathrm{Mi}$ - mosa scabrella (Bentham), bracatinga, que es una especie nativa, pionera, perteneciente a la familia Fabaceae. A lo largo de los años, especies de sucesión secundaria van reemplazando la bracatinga hasta que después de 11 años la 
población tiene una característica de bosque secundario, con predominio de varias especies secundarias (Silva et al. 2016).

Para Silva (2013), los bracatingais son apenas una de las etapas del proceso de sucesión vegetal. Este autor constató que en los bracatingais a los 4 años ya se identifican otras especies secundarias, las cuales, a partir de los 11 años superan la bracatinga y pasan a ser dominantes hasta que a los 19 años los bracatingais se transforman en bosque con estructura diamétrica secundaria. La familia Lauraceae acaba por ser la más importante, seguida de Bignoniaceae y Asteraceae, entre tanto, los géneros Ocotea y Jacaranda constituyen los más importantes con destaque para las especies Ocotea puberula (Rich.) Ness y Jacaranda micrantha Cham. por presentar el mayor valor de importancia después de la bracatinga.

Debido a que en la región metropolitana de Curitiba la bracatinga es explotada para producción de madera, principalmente para leña y carbón, además de otros usos, estos bracatingais son de gran importancia social y económica a nivel local y se constituye como un "ahorro verde" para los pequeños agricultores.

Por ser los bosques sistemas biológicos dinámicos, es necesario cuantificar sus cambios para auxiliar la toma de decisiones sobre su manejo. Respecto a eso, la modelación matemática ha jugado un rol fundamental en el manejo forestal (Burkhart y Tomé 2012).

El volumen de madera de rodales forestales es obtenido, generalmente, por la sumatoria de los volúmenes estimados a través de ecuaciones para árboles individuales localizados en las parcelas experimentales y expresado por unidad de área (hectárea). Sin embargo, la posibilidad de obtener el volumen del rodal sin utilizar ecuaciones individuales ha sido un avance en el proceso de cuantificación de los bosques (Soares y Campos 2000).

Para Scolforo (2005), la modelación de ecuaciones para el rodal (modelación por unidad de área) es una alternativa al uso de modelos para los árboles individuales y posee el objetivo de reducir trabajo y costos en los inventarios forestales. Según Figueiredo Filho et al. (1982), la aplicación de modelos matemáticos para estimar el volumen del rodal solo se justifica cuando las variables independientes del modelo son fáciles de medir en el campo y por lo tanto, trae una reducción de mano de obra y costos sin que implique pérdida significativa de precisión.

Las ecuaciones de volumen para rodales son funcionalmente similares a las ecuaciones de volumen para árboles individuales, sin embargo, en ellas son usadas variables independientes relativas a la unidad de área, tales como área basal, altura media de los árboles, media de la altura dominante, media de DAP, diámetro medio cuadrático y el número de árboles (Scolforo 2005).

Entre las investigaciones sobre modelación del volumen por unidad de área, Rosot et al.(1990) apuntan varios autores internacionales que han iniciado y desarrollado la modelación por unidad de área.
En la literatura forestal brasileña también se puede mencionar el trabajo de Figueiredo Filho et al. (1982), Silva (1985), Rosot et al. (1990), Machado y Pizatto (1998), Oliveira et al. (2005) y Machado et al. (2008). Recientemente, Cysneiros et al. (2016) modelaron el volumen comercial por hectárea en un bosque tropical en el suroeste de la Amazonia.

En estudio realizado por Machado et al. (2008), modelaron el volumen de leña por unidad de área para bracatinga en la región metropolitana de Curitiba. En dicha investigación fue utilizado un total de 29 modelos matemáticos de la literatura forestal y más una ecuación aritmética y otra logarítmica obtenida por proceso de selección de variables, con una base de datos de 229 parcelas distribuidas entre las edades de 3 y 18 años. Urbano et al. (2017) hicieron nuevamente ese levantamiento y modelaron el volumen de leña por unidad de área para bracatinga en la región metropolitana de Curitiba.

En el trabajo realizado por Urbano et al. (2017) fue adoptado el mismo procedimiento aplicado por Machado et al. (2008), pero usando una base de datos más ampliada para 320 parcelas con edades entre 3 y 20 años y utilizando una metodología actualizada de cálculo del volumen individual y de clasificación de sitio.

Otros trabajos más recientes con bracatinga se ocupan de la modelación por unidad de área para otras variables del rodal, como es el caso del estudio realizado por Ribeiro et al. (2012) que modelaron macronutrientes y micronutrientes para bracatinga, Souza et al. (2013) y (2014) que modelaron carbono orgánico total y carbono de la leña, y biomasa total y biomasa de la leña, respectivamente, para bracatingais nativos de la región metropolitana de Curitiba.

Sin embargo, para las especies secundarias presentes en los bracatingais de dicha región no se conocen modelos específicos para estimar su volumen de madera aprovechable, siendo esto una necesidad para los pequeños propietarios y para los profesionales responsables del proceso de cuantificación del volumen de leña de los bracatingais.

Por lo tanto, considerando la hipótesis de que es posible ajustar una ecuación utilizando variables del rodal que estime con precisión y confiabilidad el volumen de leña por hectárea y que resulte en menor costo en los inventarios forestales, el objetivo de este estudio es seleccionar una ecuación que resulte en mejores estimaciones del volumen de leña por hectárea de las especies secundarias que se encuentran en los bracatingais nativos de la región metropolitana de Curitiba, Paraná. Para eso, se comparó la calidad de ajuste de 29 modelos matemáticos de la literatura y otros dos modelos desarrollados por medio del método de selección de variables hacia adelante paso a paso "forward stepwise", identificando la mejor ecuación en términos del menor error de estimativa, alta precisión y facilidad de uso, para que sea aplicada por profesionales que realizan inventarios forestales en estos bracatingais. 


\section{MÉTODOS}

Caracterización del área de estudio. Para el desarrollo de la presente investigación se utilizaron datos de bracatingais nativos de la región metropolitana de Curitiba, colectados en catorce municipios. De acuerdo con Mazza et al. (2000), en esta región los bracatingais ocupan una superficie aproximada de 66.000 hectáreas, cerca del 9,4\% del área territorial de los municipios.

La bracatinga forma parte del proceso natural de sucesión del bosque ombrófilo mixto (bosque con araucaria) y en los bracatingais está asociada con otras 153 otras especies. La lista con estas especies puede ser observada en el trabajo de Silva (2013) que estudió la estructura y la florística de bracatingais con enfoque sucesional.

El área de estudio se encuentra en el primer altiplano del Estado de Paraná, Brasil, el cual presenta clima templado, mesotérmico húmedo sin estación seca, con cuatro estaciones bien diferenciadas, veranos cálidos y lluviosos e inviernos fríos con períodos secos ocasionales, que de acuerdo con el sistema de clasificación de Köppen es considerado como clima Cfb (Caviglione et al. 2000). La temperatura media anual es alrededor de $17^{\circ} \mathrm{C}$, con mínima de $11{ }^{\circ} \mathrm{C}$ y máxima $24^{\circ} \mathrm{C}$. La precipitación anual oscila entre 1.400 y $1.600 \mathrm{~mm}$, con una ligera disminución en el invierno, sin déficit hídrico.

Las coordenadas geográficas de la zona de estudio limitan al norte por la latitud $24^{\circ} 58^{\prime} 11^{\prime \prime} \mathrm{S}$, al sur por la latitud $25^{\circ} 55^{\prime} 44^{\prime \prime} \mathrm{S}$, al oeste por la longitud $49^{\circ} 29^{\prime} 09^{\prime \prime} \mathrm{W}$ y al este por la longitud $49^{\circ} 03$ ' $58^{\prime \prime}$ W (Silva et al. 2016).

Según Souza et al. (2013), el área de estudio tiene en las regiones este, norte y sur una fuerte influencia de la Sierra del Mar, y la región más al oeste, presenta influencia de la Escarpa Devoniana. En la región norte de esta, donde el terreno es más accidentado, la altitud varía de $850 \mathrm{~m}$ en Agudos do Sul para 980 m en Bocaiúva do Sul.

Base de datos. Los datos para la modelación del volumen del rodal provienen de otros estudios según lo descrito por Silva et al. (2016). Se componen de 320 parcelas temporales distribuidas aleatoriamente en toda el área de estudio, en bracatingais con edades comprendidas entre 3 y 20 años, no siendo encontrados bracatingais a la edad de 16 años.

La edad de los árboles de bracatinga en las parcelas fue determinada por información local (propietarios) y verificada, cuando necesario, mediante la lectura de los anillos de crecimiento del tronco (Silva et al. 2016).

En cada parcela y para todos los árboles, fueron medidos el diámetro a 1,30 m sobre el nivel del suelo (DAP) y la altura total. Se destaca que del total de parcelas utilizadas, 219 de ellas tienen especies de composición mixta (bracatinga + otras especies secundarias), 81 solo poseen bracatinga, mientras que en 20 parcelas no está presente dicha especie.Las unidades de la muestra por edad se presentan en el cuadro 1.
En la muestra utilizada hay parcelas de diferentes tamaños, pero los valores de las variables de cada parcela se extrapolaron, por regla de tres simples, a nivel de hectárea con el fin de estandarizarlas. Los datos de cada parcela fueron preparados de la siguiente forma para cumplir con los objetivos propuestos y desarrollar el proceso de modelación: cálculo de la altura media $(\overline{\mathrm{h}})$, diámetros medios $(\overline{\mathrm{d}})$ y el diámetro medio cuadrático ( $\mathrm{dg}$ ) de árboles en las parcelas; determinación de alturas dominantes $\left(\mathrm{h}_{\mathrm{dom}}\right)$ de cada parcela con posterior clasificación de sitio; cálculo del volumen de madera individual de cada árbol con posterior extrapolación a nivel de hectárea; cálculo del área transversal de cada árbol y su extrapolación a nivel de hectárea (área basal por hectárea).

Los valores observados de altura dominante $-\mathrm{h}_{\text {dom }}$ de bracatinga en cada parcela se determinaron con base en el concepto de Assmann, es decir, la altura media de los 100 árboles más gruesos por hectárea. Para clasificar las parcelas en sus clases de productividad se utilizó la ecuación 1, que es la mejor ecuación de sitio ajustada por Machado

Cuadro 1. Intensidad de muestreo, por edad, en los bracatingais. Sample intensity, by age, in the bracatinga forests.

\begin{tabular}{|c|c|c|c|c|c|}
\hline $\begin{array}{c}\text { Edad } \\
\text { (años) }\end{array}$ & $\begin{array}{l}\text { Número } \\
\text { total de } \\
\text { parcelas }\end{array}$ & $\begin{array}{l}\text { Cantidad de } \\
\text { parcelas con } \\
\text { bracatinga }\end{array}$ & $\begin{array}{l}\text { Número de } \\
\text { parcelas con } \\
\text { secundarias }\end{array}$ & $\begin{array}{c}\text { Área } \\
\text { muestreada } \\
\left(\mathrm{m}^{2}\right)\end{array}$ & $\begin{array}{c}\text { Área de } \\
\text { cada parcela } \\
\left(\mathrm{m}^{2}\right)\end{array}$ \\
\hline 3 & 8 & 8 & 0 & 800 & 100 \\
\hline 4 & 21 & 21 & 5 & 4.920 & 234 \\
\hline 5 & 27 & 27 & 6 & 6.315 & 234 \\
\hline 6 & 32 & 32 & 18 & 8.125 & 254 \\
\hline 7 & 36 & 36 & 24 & 9.610 & 267 \\
\hline 8 & 22 & 22 & 17 & 5.800 & 264 \\
\hline 9 & 25 & 25 & 22 & 6.720 & 269 \\
\hline 10 & 16 & 16 & 16 & 4.800 & 300 \\
\hline 11 & 18 & 18 & 18 & 5.720 & 318 \\
\hline 12 & 15 & 15 & 15 & 4.270 & 285 \\
\hline 13 & 19 & 19 & 18 & 6.000 & 316 \\
\hline 14 & 21 & 21 & 21 & 6.900 & 329 \\
\hline 15 & 13 & 13 & 12 & 5.000 & 385 \\
\hline 17 & 6 & 6 & 6 & 3.400 & 567 \\
\hline 18 & 9 & 9 & 9 & 2.980 & 331 \\
\hline 19 & 29 & 11 & 29 & 5.800 & 200 \\
\hline 20 & 3 & 1 & 3 & 900 & 300 \\
\hline Total & 320 & 300 & 239 & 88.060 & - \\
\hline
\end{tabular}


et al. (2011) para bracatingais nativos de la región metropolitana de Curitiba.

$$
h_{\text {dom }}=\frac{S^{*}\left[1-\exp \left(-0,2285^{*} E\right)\right]^{1,1438}}{\left[1-\exp \left(-0,2285^{*} E_{\text {ref }}\right)\right]^{1,1438}}
$$

Donde: $\mathrm{h}_{\mathrm{dom}}=$ altura dominante $(\mathrm{m}) ; \mathrm{S}=$ límite de la curva de sitio en la edad de referencia; $\mathrm{E}=$ edad (años); $\mathrm{E}_{\mathrm{ref}}=$ edad de referencia (7 años).

Los valores resultantes de la estimación establece tres clases de sitio a los siete años, I, II y III, con índices de sitio de 17,13 y $9 \mathrm{~m}$, respectivamente.

La clasificación de sitios resultó en 48 parcelas en la clase III, 190 en la clase II y 62 en la clase I, sumando 300 parcelas con presencia de bracatinga. Sin embargo, debido a la existencia de 20 parcelas únicamente con especies secundarias, fue necesario clasificar su clase de sitio de acuerdo con la proximidad a las demás. Este procedimiento afectó el error de muestreo en $+0,4 \%$, aumentando la heterogeneidad de los datos volumétricos, como se esperaba, por tratarse de especies nativas secundarias.

Cálculo de volúmenes individuales (volumen de leña con corteza) de los árboles de las parcelas. Para la estimación de los volúmenes individuales de los troncos de especies secundarias se utilizaron ecuaciones volumétricas ajustadas por Santos et al. (2006) para latifoliadas presentes en los bosques ombrófilos mixtos en el estado de Paraná. Para los árboles con dap inferiores a $15 \mathrm{~cm}$ se usó la ecuación 2 y para los árboles con dap mayores o iguales a $15 \mathrm{~cm}$ se usó la ecuación 3.

$$
\begin{gathered}
\ln (\mathrm{v})=-8,87590982+1,892218745 * \ln (\mathrm{d})+ \\
0,739037687 * \ln (\mathrm{h}) \\
\mathrm{v}=-0,01295354+0,00007636 * \mathrm{~d}^{2} \mathrm{~h}- \\
0,000102192 * \mathrm{dh}^{2}+0,001340984 * \mathrm{~h}^{2}
\end{gathered}
$$

Donde: $\ln =$ logaritmo neperiano; $\mathrm{v}=$ volumen del fuste $\left(\mathrm{m}^{3}\right) ; \mathrm{d}=$ diámetro a la altura del pecho $(\mathrm{cm}) ; \mathrm{h}=$ altura total del árbol (m).

La ecuación 2 presenta un $\mathrm{R}^{2}=0,961$ con $\mathrm{Syx} \%=14,69$ y la ecuación 3 presenta $\mathrm{R}^{2}=0,973$ con $\mathrm{Syx} \%=8,8$. Siendo $\mathrm{R} 2$ = coeficiente de determinación; Syx \% = error estándar.

En medio de los bracatingais, se encuentran algunos pocos ejemplares de araucaria (Araucaria angustifolia (Bertol.) Kuntze), conífera nativa de la región. Para estimar el volumen total de araucaria se utilizó la ecuación 4 citada por Figueiredo Filho et al. (2014), cuya expresión es:

$$
\mathrm{v}=0,03830416+0,52239325 * \mathrm{~d}^{2} \mathrm{~h}
$$

Donde: $\mathrm{v}, \mathrm{d}$ y h ya fueron definidos anteriormente.
Como no se ha encontrado en la literatura una ecuación apropiada para estimar el volumen de leña de árboles de especies secundarias, se multiplicó el volumen estimado del fuste por 1,3. Este factor de expansión del volumen considera para las especies secundarias la misma proporción de ramas observadas en árboles cubicadas y xilometradas para bracatinga descrito por Machado et al.(2006).

Detección de datos discrepantes o atípicos - "outliers". Se realizó un análisis exploratorio de la base de datos para detectar la presencia de valores atípicos de volumen por hectárea para cada edad muestreada y para la dispersión de los datos a lo largo del tiempo.

Para excluir los valores extremos en cada clase de edad, fue utilizado el método basado en la amplitud intercuartil (IQR - Interquartile Range), usando como criterio de exclusión cualquier valor mayor que 1,5 veces el rango intercuartil a partir del $3^{\circ}$ cuartil, y aquellos valores menores de 1,5 veces el rango intercuartil a partir del $1^{\circ}$ cuartil (Tukey 1977).

Modelación del volumen de leña del rodal para las especies secundarias. Para la modelación del volumen de leña del rodal de las especies secundarias presentes en los bracatingais nativos, se ajustaron los mismos modelos utilizados por Urbano et al. (2017) para bracatinga en la Región Metropolitana de Curitiba. De los 29 modelos matemáticos ajustados en dicho estudio, 12 son de naturaleza aritmética, cuatro semi-logarítmica y 13 de naturaleza logarítmica, los cuales son presentadas a continuación de acuerdo con el autor.

- Stoate (1945)

$$
\begin{gathered}
V=b 0+b 1 \cdot G \bar{h}+b 2 \cdot G+b 3 \cdot \bar{h} \\
V=b 0+b 1 \cdot G h_{d o m}+b 2 \cdot G+b 3 \cdot h_{d o m}
\end{gathered}
$$

- Spurr (1952)

$$
\begin{gathered}
V=b 0+b 1 \cdot G \bar{h} \\
V=b 0+b 1 \cdot G h_{d o m} \\
V=b 0+b 1 \cdot G \\
V=b 0+b 1 \cdot G^{2}+b 2 \cdot G \\
\ln (V)=b 0+b 1 \cdot \ln (G \bar{h}) \\
\ln (V)=b 0+b 1 \cdot \ln (G) \\
\ln (V)=b 0+b 1 \cdot \ln \left(G^{2}\right) \\
\ln (V)=b 0+b 1 \cdot \ln \left(G^{2} h_{d o m}\right)
\end{gathered}
$$




$$
\ln (\mathrm{V})=\mathrm{b} 0+\mathrm{b} 1 \cdot \ln \left(\mathrm{Gh}_{\mathrm{dom}}\right)
$$

- Takata (1959)

$$
\mathrm{V}=\mathrm{G} \overline{\mathrm{h}} / \mathrm{b} 0+\mathrm{b} 1 \cdot \mathrm{d}_{\mathrm{g}}
$$

- Machado (1973)

$$
\mathrm{V}=\mathrm{b} 0+\mathrm{b} 1 . \mathrm{G}^{2}
$$

- Silva (1979)

$$
\mathrm{V}=\mathrm{b} 0+\mathrm{b} 1 \cdot \mathrm{G}^{2} \mathrm{~h}_{\mathrm{dom}}
$$

- Figueiredo Filho (1982)

$$
\begin{gathered}
V=b 0+b 1 \cdot \ln (G \bar{h}) \\
V=b 0+b 1 \cdot \ln \left(G^{2} \bar{h}\right) \\
V=b 0+b 1 \cdot \ln (G) \\
V=b 0+b 1 \cdot \ln \left(G^{2}\right) \\
\ln (V)=b 0+b 1 \cdot \ln \left(G^{2} \bar{h}\right)
\end{gathered}
$$

- Rosot (1990)

$$
\begin{gathered}
V=b 0+b 1 \cdot G^{2} \bar{h} \\
\ln (V)=b 0+b 1 \cdot \ln (G N) \\
\ln (V)=b 0+b 1 \cdot \ln \left(\bar{d}^{2} \bar{h}\right)
\end{gathered}
$$

- Ung y Ouellett (1991)

$$
\begin{gathered}
V=b 0 \cdot G^{b 1} \cdot h_{d o m}{ }^{b 2} \\
V=b 0 \cdot G^{b 1} \cdot \bar{h}^{b 2} \\
\ln (V)=b 0+\ln (G \bar{h})^{b 1}+\ln \left(G_{d o m}\right)^{b 2} \\
\ln (V)=b 0+b 1 \cdot \ln (G)+b 2 \cdot \ln (\bar{h}) \\
\ln (V)=b 0+b 1 \cdot \ln (G \bar{h})+b 2 \cdot \ln \left(G_{d o m}\right)
\end{gathered}
$$

- Scolforo (1997)

$$
\begin{aligned}
& \ln (\mathrm{V})=\mathrm{b} 0+\mathrm{b} 1 \cdot \ln \left(\mathrm{G}^{2} \mathrm{~N}\right) \\
& \ln (\mathrm{V})=\mathrm{b} 0+\mathrm{b} 1 \cdot \ln (\mathrm{N} \overline{\mathrm{d}})
\end{aligned}
$$

Donde: $\mathrm{V}=$ volumen de la leña con corteza por hectárea $\left(\mathrm{m}^{3} / \mathrm{ha}\right) ; \mathrm{G}=$ área basal $\left(\mathrm{m}^{2} / \mathrm{ha}\right) ;$ = altura total media $(\mathrm{m})$; $\mathrm{h}_{\mathrm{dom}}=$ altura dominante $(\mathrm{m})$; dg = diámetro medio cuadrá- tico $(\mathrm{cm})$; = diámetro medio a la altura del pecho $(\mathrm{cm})$; $\mathrm{N}$ = número de árboles por hectárea; $\ln =$ logaritmo natu$\mathrm{ral} ; \mathrm{b}_{\mathrm{i}}=$ coeficientes de los modelos.

En el ajuste de los modelos lineales aritméticos o logarítmicos, se utilizó la técnica de mínimos cuadrados por regresión lineal simple o múltiple. En el caso de los modelos no lineales, fueron ajustados por regresión no lineal. También se ajustaron otros modelos considerando la correlación de las variables por medio del método "forward stepwise", el cuales un procedimiento de selección de variables basado en la inclusión paso a paso de las mismas, donde el algoritmo comienza con el intercepto y luego incorpora secuencialmente en el modelo una a una las variables predictoras que mejora significativamente el ajuste.

Análisis de los modelos ajustados. Para que la ecuación elegida tenga sus estimaciones confiables, su selección debe ser con base en los siguientes criterios estadísticos: mayor coeficiente de determinación $\left(\mathrm{R}^{2}\right)$, menor error estándar de la estimación en porcentaje (Syx\%), ausencia de multicolinealidad y análisis gráfico de residuos para verificar heterocedasticidad y sesgos presentes en la estimación.

El diagnóstico de multicolinealidad fue realizado a través de la observación de la no significancia $(P<0,05)$ de los coeficientes de las variables independientes utilizadas en modelos múltiples de regresión lineal, conforme propuesto por Scolforo (2005).

Para las ecuaciones logarítmicas ajustadas, los valores estimados fueron corregidos para eliminar la discrepancia logarítmica y hacerlos comparables con las ecuaciones aritméticas. En este caso, los volúmenes estimados fueron multiplicados por el factor de corrección de Mayer - Fcm $\mathrm{y}$, consecuentemente, el $\mathrm{R}^{2}$ y Syx fueron recalculados:

$$
\mathrm{Fcm}=e^{0,5 * S y x^{2}}
$$

Donde $e=$ base de los logaritmos naturales y Syx $=$ error estándar de la estimación en unidades logarítmicas

Después de elegir la mejor ecuación, se verificó el valor de la prueba de Durbin Watson para verificar si los residuos se correlacionan y posteriormente fue realizada su validación mediante la prueba de adherencia de chicuadrado $\left(\mathrm{X}^{2}\right)$ con $P<0,05$, y el análisis gráfico de los residuos. En la selección de las parcelas para validación, fue acogida la recomendación dada por Prodan et al. (1997), en relación a que los datos utilizados para tal fin deben ser independientes de los datos utilizados en el ajuste, cubriendo las distintas edades, clases de sitio y regímenes de manejo. 


\section{RESULTADOS}

Análisis de los datos discrepantes. Como resultado de análisis del volumen por hectárea, se retiraron ocho parcelas discrepantes que contenían las especies secundarias. En consecuencia, de las 239 parcelas inicialmente contempladas, quedaron 231 parcelas con especies secundarias cuya información fue utilizada en los procesos de modelación volumétrica de los rodales muestreados.

Correlación entre las variables utilizadas en el ajuste. Después de la exclusión de las parcelas con valores atípicos, se hizo el análisis de correlación lineal simple (cuadro 2) entre las variables independientes (medidas, calculadas, combinadas y transformadas) y las variables dependientes (volumen de leña por hectárea en la forma aritmética y logarítmica). El nivel de significancia utilizado en todos los análisis para los coeficientes de correlación fue de $5 \%$.

El área basal $(G)$, que es una variable muy importante para evaluar el volumen por unidad de superficie debido a su facilidad de medición y el alto grado de correlación con el volumen del rodal, mostró una buena correlación con el volumen de leña $(0,974$ y 0,799$)$, en la forma aritmética y logarítmica, respectivamente.

Las combinaciones de área basal con las variables altura media y altura dominante fueron las que resultaron en

Cuadro 2. Coeficiente de correlación lineal simple entre las variables independientes y dependientes.

Simple linear correlation coefficient between independent and dependent variables.

\begin{tabular}{|c|c|c|c|c|c|c|c|c|}
\hline \multirow{2}{*}{$\begin{array}{c}\text { Variables } \\
\text { Independientes }\end{array}$} & \multicolumn{2}{|c|}{$\begin{array}{c}\text { Variables } \\
\text { dependientes }\end{array}$} & \multirow{2}{*}{$\begin{array}{c}\text { Variables } \\
\text { independientes }\end{array}$} & \multicolumn{2}{|c|}{$\begin{array}{c}\text { Variables } \\
\text { dependientes }\end{array}$} & \multirow{2}{*}{$\begin{array}{c}\text { Variables } \\
\text { independientes }\end{array}$} & \multicolumn{2}{|c|}{$\begin{array}{l}\text { Variables } \\
\text { dependientes }\end{array}$} \\
\hline & $\mathrm{V}$ & $\ln (\mathrm{V})$ & & $\mathrm{V}$ & $\ln (\mathrm{V})$ & & V & $\ln (\mathrm{V})$ \\
\hline I & 0,729 & 0,794 & GN & 0,833 & 0,621 & $\ln \left(\mathrm{G}^{2} \mathrm{~N}\right)$ & 0,749 & 0,989 \\
\hline $\mathrm{N}$ & 0,714 & 0,806 & $\mathrm{G}^{2} \mathrm{~N}$ & 0,717 & 0,417 & $\ln \left(\overline{\mathrm{d}}^{2}\right)$ & 0,734 & 0,670 \\
\hline$\overline{\mathrm{d}}$ & 0,757 & 0,645 & $\ln (\overline{\mathrm{d}})$ & 0,779 & 0,716 & $\ln (\mathrm{N})$ & 0,716 & 0,978 \\
\hline$\overline{\mathrm{h}}$ & 0,310 & 0,273 & $\ln (\overline{\mathrm{h}})$ & 0,270 & 0,235 & $1 / \overline{\mathrm{d}}$ & $-0,769$ & $-0,761$ \\
\hline 'dg & 0,780 & 0,655 & $\ln \left(\mathrm{h}_{\mathrm{dom}}\right)$ & $-0,058$ & 0,091 & $1 / \overline{\mathrm{h}}$ & $-0,224$ & $-0,186$ \\
\hline $\mathrm{S}$ & $-0,359$ & $-0,300$ & $\ln (\mathrm{dg})$ & 0,801 & 0,736 & $1 / h_{d o m}$ & 0,074 & $-0,039$ \\
\hline $\mathrm{h}_{\mathrm{dom}}$ & $-0,033$ & 0,135 & $\ln (G)$ & 0,783 & 0,996 & $1 / \mathrm{dg}$ & $-0,786$ & $-0,786$ \\
\hline G & 0,974 & 0,799 & $\ln (\overline{\mathrm{d}} \overline{\mathrm{h}})$ & 0,663 & 0,603 & $1 / \mathrm{G}$ & $-0,402$ & $-0,778$ \\
\hline$\overline{\mathrm{d}} \overline{\mathrm{h}}$ & 0,625 & 0,518 & $\ln \left(\overline{\mathrm{d}}^{2} \overline{\mathrm{h}}\right)$ & 0,734 & 0,670 & $1 / \overline{\mathrm{d}} \overline{\mathrm{h}}$ & $-0,620$ & $-0,605$ \\
\hline$\overline{\mathrm{d}}^{2} \overline{\mathrm{h}}$ & 0,606 & 0,463 & $\ln \left(\overline{\mathrm{d}} \mathrm{h}_{\mathrm{dom}}\right)$ & 0,544 & 0,607 & $1 / \overline{\mathrm{d}}^{2} \overline{\mathrm{h}}$ & $-0,679$ & $-0,713$ \\
\hline$\overline{\mathrm{d}} \mathrm{h}_{\mathrm{dom}}$ & 0,582 & 0,612 & $\ln \left(\overline{\mathrm{d}}^{2} \mathrm{~h}_{\mathrm{dom}}\right)$ & 0,704 & 0,714 & $1 / \overline{\mathrm{d}} \mathrm{h}_{\mathrm{dom}}$ & $-0,462$ & $-0,543$ \\
\hline$\overline{\mathrm{d}}^{2} \mathrm{~h}_{\mathrm{dom}}$ & 0,690 & 0,606 & $\ln \left(G^{2}\right)$ & 0,783 & 0,996 & $1 / \overline{\mathrm{d}}^{2} \mathrm{~h}_{\mathrm{dom}}$ & $-0,609$ & $-0,705$ \\
\hline $\mathrm{G}^{2}$ & 0,860 & 0,534 & $\ln (G \bar{h})$ & 0,796 & 0,999 & $1 / \mathrm{G}^{2}$ & $-0,227$ & $-0,530$ \\
\hline $\mathrm{G}_{\overline{\mathrm{h}}}$ & 0,989 & 0,796 & $\ln \left(G^{2} \bar{h}\right)$ & 0,791 & 0,999 & $1 / \mathrm{G} \overline{\mathrm{h}}$ & $-0,417$ & $-0,800$ \\
\hline $\mathrm{G}^{2} \overline{\mathrm{h}}$ & 0,895 & 0,553 & $\ln \left(\mathrm{Gh}_{\mathrm{dom}}\right)$ & 0,758 & 0,989 & $1 / \mathrm{G}^{2} \overline{\mathrm{h}}$ & $-0,237$ & $-0,549$ \\
\hline $\mathrm{G}_{\mathrm{hdo}} \mathrm{m}$ & 0,947 & 0,809 & $\ln \left(\mathrm{G}^{2} \mathrm{~h}_{\mathrm{dom}}\right)$ & 0,772 & 0,995 & $1 / \mathrm{Gh}_{\mathrm{dom}}$ & $-0,372$ & $-0,741$ \\
\hline $\mathrm{G}^{2} \mathrm{~h}_{\mathrm{dom}}$ & 0,868 & 0,549 & $\ln (\operatorname{dg} \bar{h})$ & 0,705 & 0,641 & $1 / \mathrm{G}^{2} \mathrm{~h}_{\mathrm{dom}}$ & $-0,217$ & $-0,513$ \\
\hline $\operatorname{dg} \overline{\mathrm{h}}$ & 0,662 & 0,542 & $\ln \left(\mathrm{dg}^{2} \overline{\mathrm{h}}\right)$ & 0,768 & 0,701 & $1 / \mathrm{dg} \overline{\mathrm{h}}$ & $-0,662$ & $-0,655$ \\
\hline $\mathrm{dg}^{2} \overline{\mathrm{h}}$ & 0,635 & 0,474 & $\ln \left(\operatorname{dgh}_{\mathrm{dom}}\right)$ & 0,604 & 0,654 & $1 / \mathrm{dg}^{2} \overline{\mathrm{h}}$ & $-0,703$ & $-0,755$ \\
\hline $\mathrm{dgh}_{\mathrm{dom}}$ & 0,642 & 0,643 & $\ln \left(\mathrm{dg}^{2} \mathrm{~h}_{\mathrm{dom}}\right)$ & 0,744 & 0,743 & $1 / \mathrm{dgh}_{\mathrm{dom}}$ & $-0,519$ & $-0,604$ \\
\hline $\mathrm{dg}^{2} \mathrm{~h}_{\mathrm{dom}}$ & 0,719 & 0,604 & $\ln (\mathrm{GN})$ & 0,727 & 0,981 & $1 / \mathrm{dg}^{2} \mathrm{~h}_{\mathrm{dom}}$ & $-0,643$ & $-0,754$ \\
\hline
\end{tabular}

Donde: $\mathrm{V}=$ volumen de leña con corteza en $\mathrm{m}^{3} / \mathrm{ha} ; \ln (\mathrm{V})=$ logaritmo natural del volumen de leña con corteza; $\mathrm{I}=$ edad (años); $\mathrm{N}=$ número de árboles por hectárea; = media del diametro a la altura del pecho $(\mathrm{cm}) ;=$ media de la altura total $(\mathrm{m}) ; \mathrm{dg}=$ diámetro medio cuadrático $(\mathrm{cm}) ; \mathrm{S}=\mathrm{sitio}(\mathrm{m}) ; \mathrm{h}_{\text {dom }}$ $=$ altura dominante $(\mathrm{m}) ; \mathrm{y} \mathrm{G}=$ área basal por hectárea $\left(\mathrm{m}^{2}\right)$. 
correlaciones más altas, tanto con el volumen en la forma aritmética como en la forma logarítmica. La mayor correlación lineal simple (r) del volumen de leña con corteza por hectárea $(\mathrm{V})$ fue con la variable combinada $(\mathrm{G})$, con $\mathrm{r}=0,989$, seguido de la variable $\mathrm{G}$, con $\mathrm{r}=0,974$ y la variable $\left(\mathrm{Gh}_{\mathrm{dom}}\right)$, con $\mathrm{r}=0,947$. En el caso de la variable $\ln (\mathrm{V})$ las mayores correlaciones fueron obtenidas con las variables $\ln (G)$ y $\ln (G)$, que mostraron $r=0,999$, seguido por variables $\ln (\mathrm{G})$ y $\ln \left(\mathrm{G}^{2}\right)$, que mostraron $r=0,996$.

Estadística descriptiva de las principales variables utilizadas. Posterior a la eliminación de valores atípicos, se calculó con la información de las 231 parcelas, los valores mínimo, medio y máximo así como la desviación estándar y el coeficiente de variación en porcentaje (CV\%) de las principales variables utilizadas en la modelación del volumen del rodal de las especies secundarias (cuadro 3).

Ajuste de los modelos. En la modelación del volumen de la leña para las especies secundarias se utilizaron 200 parcelas para el ajuste de las ecuaciones y 31 parcelas para la validación de la ecuación que presentó mejores parámetros de ajuste. En el cuadro 4 se presentan los coeficientes y estadísticas de ajuste y precisión para cada ecuación ajustada, ordenados de acuerdo con el error estándar.

Se puede observar (cuadro 4) que el error estándar estimado (Syx) varió de 12,94 \% a 71,6 \%, y el coeficiente de determinación ajustado ( $\mathrm{R}^{2}$ aj.) varió de 0,987 a 0,611.

Entre los modelos probados y ajustados, se destacan el modelo 28 no lineal de Ung y Ouellett (1991) con Syx = $12,94 \%$ y R $\mathrm{R}^{2}$ aj $=0,987$, seguido por la ecuación ajustada para el modelo logaritmo 30 de Ung y Ouellett (1991), con $\operatorname{Syx}=13,10 \%$ y R ${ }^{2} a j=0,987$, y la ecuación ajustada para el modelo 5 de Stoate (1945), con Syx $=13,27 \%$ y $\mathrm{R}^{2} \mathrm{aj}=0,987$. Sin embargo, la ecuación ajustada para el modelo 5 presenta un problema de multicolinealidad entre las variables que lo componen. La distribución gráfica de los residuos de las ecuaciones 28 y 30 se muestran en la figura 1, más adelante junto con los gráficos de los residuos para las ecuaciones ajustadas a través del proceso de selección de variable "forward stepwise".

En esta investigación también han sido ajustadas una ecuación aritmética y otra logarítmica por el proceso de selección "forward stepwise", que fueron nombrados como ecuaciones 35 y 36, respectivamente. Estas ecuaciones son presentadas a continuación. Sus coeficientes y estadísticas de ajuste y precisión se muestran en el cuadro 5, y la distribución gráfica de residuos se muestra en la figura 1 .

$$
\begin{gathered}
V=b 0-b 1 \cdot \bar{d}^{2} \bar{h}+b 2 \cdot G^{2}+b 3 \cdot G \bar{h}-b 4 \cdot G^{2} \bar{h}+b 5 \cdot d^{2} \bar{h}[35] \\
\ln (V)=b 0-b 1 \cdot \bar{d}+b 2 \cdot \ln \left(G^{2}\right)-b 3 \cdot \ln (N \cdot \bar{d})-b 4 \cdot \bar{h}^{-1}[36]
\end{gathered}
$$

Conforme los resultados, la ecuación seleccionada para estimar el volumen por hectárea de todas las especies secundarias es la ecuación 36, mismo permaneciendo en segundo lugar en el ranking (con error estándar 1,19\% mayor que el primer lugar), justificado porque presenta mejor distribución de los residuos sin sesgo y heterocedasticidad, y es también un modelo sin multicolinealidad. Para la ecuación 36 se verificó el valor de la prueba de autocorrelación de los residuos (Durbin Watson), donde el valor calculado de 1,87 fue mayor que el límite superior tabulado de 1,81 indicando que los residuos no están correlacionados. Por lo tanto, se utilizó esta ecuación para la prueba de validación.

La prueba de adhesión Chi-cuadrado $\left(\aleph^{2}\right)$ utilizado para la validación de la ecuación presentó un $\aleph^{2}$ cal $=2,64$ menor que $\aleph^{2} \operatorname{tab}(\alpha=5 \%)=43,77$ siendo no significativo; por consiguiente se acepta la hipótesis nula e se determina que

Cuadro 3. Estadísticas descriptivas de las principales variables utilizadas para el ajuste de los modelos.

\begin{tabular}{|c|c|c|c|c|c|}
\hline Variables & Mínimo & Media & Máximo & Desviación estándar & $\mathrm{CV} \%$ \\
\hline Edad (años)* & 4 & 11,71 & 20 & 4,41 & 37,66 \\
\hline Número de árboles por hectárea- $\mathrm{N}$ & 37 & 1103 & 4200 & 906 & 82,17 \\
\hline Media del diámetro a la altura del pecho - $(\mathrm{cm})$ & 5,05 & 7,32 & 18,43 & 2,01 & 27,45 \\
\hline Media de la altura total- $(\mathrm{m})$ & 3,14 & 6,63 & 12,05 & 1,23 & 18,62 \\
\hline Diámetro medio cuadrático - $\mathrm{dg}(\mathrm{cm})$ & 5,05 & 7,68 & 20,59 & 2,46 & 32,03 \\
\hline Sitio - S (m)* & 9,00 & 13,00 & 17,00 & 2,66 & 20,94 \\
\hline Altura dominante $-\mathrm{h}_{\mathrm{dom}}(\mathrm{m})^{*}$ & 5,00 & 14,19 & 21,33 & 2,91 & 20,49 \\
\hline Área basal por hectárea - G $\left(\mathrm{m}^{2}\right)$ & 0,08 & 6,33 & 44,02 & 7,02 & 110,98 \\
\hline Volumen por hectárea- V $\left(\mathrm{m}^{3}\right)$ & 0,59 & 52,06 & 331,10 & 59,87 & 115,01 \\
\hline
\end{tabular}

Descriptive statistics of the main variables used for fit of models.

* De la especie Mimosa scabrella (bracatinga). 
Cuadro 4. Coeficiente de las ecuaciones ajustadas y sus respectivas estadísticas de ajuste y precisión. Coefficients of the fitted equations and their respective adjustment and precision statistics.

\begin{tabular}{|c|c|c|c|c|c|c|c|c|}
\hline Ecuación & b0 & $\mathrm{b} 1$ & $\mathrm{~b} 2$ & b3 & FCM & $\mathrm{R}^{2} \mathrm{aj}$ & Syx & Syx \% \\
\hline 28 & $2,2324^{* *}$ & $1,0123^{* *}$ & $0,6683^{* *}$ & --- & --- & 0,987 & 6,933 & 12,94 \\
\hline 30 & $0,7096^{* *}$ & $1,0103^{* *}$ & $0,7138^{* *}$ & --- & 1,0011 & 0,987 & 7,021 & 13,10 \\
\hline 5 & $-3,4013^{\mathrm{ns}}$ & $0,7815^{* *}$ & $3,0112^{* *}$ & $0,3927^{\text {ns }}$ & --- & 0,987 & 7,111 & 13,27 \\
\hline 23 & $1,0890^{* *}$ & $0,5078^{* *}$ & ---- & ---- & 1,0018 & 0,984 & 7,700 & 14,37 \\
\hline 31 & $0,1174^{* *}$ & $0,9205^{* *}$ & $0,0817^{* *}$ & --- & 1,0024 & 0,981 & 8,471 & 15,81 \\
\hline 7 & $0,6768^{\text {ns }}$ & $1,1897^{* *}$ & --- & --- & ---- & 0,977 & 9,422 & 17,58 \\
\hline 16 & $0,8422^{* *}$ & $0,0912^{* *}$ & ---- & ---- & ---- & 0,977 & 9,426 & 17,59 \\
\hline 6 & $0,8496^{\mathrm{ns}}$ & $0,1578^{* *}$ & $6,2908^{* *}$ & $-0,1819^{\text {ns }}$ & --- & 0,950 & 13,756 & 25,67 \\
\hline 27 & $4,7351^{* *}$ & $4,7351^{* *}$ & $4,7351^{* *}$ & --- & --- & 0,949 & 13,842 & 25,83 \\
\hline 10 & $-2,5616^{\mathrm{ns}}$ & $-0,0246^{*}$ & $8,9636^{* *}$ & ---- & ---- & 0,947 & 14,217 & 26,53 \\
\hline 9 & $-0,6068^{\mathrm{ns}}$ & $8,3091^{* *}$ & ---- & ---- & --- & 0,945 & 14,366 & 26,81 \\
\hline 11 & $2,0303^{* *}$ & $1,0229^{* *}$ & --- & ---- & 1,0103 & 0,945 & 14,477 & 27,01 \\
\hline 12 & $2,0303^{* *}$ & $1,0229^{* *}$ & --- & --- & 1,0103 & 0,945 & 14,477 & 27,01 \\
\hline 13 & $2,0303^{* *}$ & $0,5114^{* *}$ & --- & --- & 1,0103 & 0,945 & 14,477 & 27,01 \\
\hline 14 & $0,7041^{* *}$ & $0,5079^{* *}$ & --- & --- & 1,0115 & 0,939 & 15,154 & 28,27 \\
\hline 29 & $-1,8177^{* *}$ & $0,3883^{* *}$ & $0,9340^{* *}$ & ---- & ---- & 0,918 & 17,561 & 32,77 \\
\hline 8 & $-0,9001^{\text {ns }}$ & $0,6020^{* *}$ & --- & ---- & --- & 0,894 & 20,029 & 37,37 \\
\hline 15 & $-0,5613^{* *}$ & $0,9974^{* *}$ & --- & --- & 1,0262 & 0,892 & 20,218 & 37,72 \\
\hline 32 & $-0,0605^{\mathrm{ns}}$ & $0,3679^{* *}$ & ---- & ---- & 1,0276 & 0,848 & 24,010 & 44,80 \\
\hline 24 & $28,6749^{* *}$ & $0,0390^{* *}$ & --- & --- & --- & 0,798 & 27,671 & 51,63 \\
\hline 25 & $-1,2033^{* *}$ & $0,5704^{* *}$ & ---- & ---- & 1,0460 & 0,773 & 29,302 & 54,67 \\
\hline 18 & $28,9949^{* *}$ & $0,0197^{* *}$ & ---- & ---- & ---- & 0,749 & 30,797 & 57,46 \\
\hline 17 & $30,2695^{* *}$ & $0,2479^{* *}$ & --- & ---- & ---- & 0,735 & 31,640 & 59,04 \\
\hline 33 & $-6,7044^{* *}$ & $1,1591^{* *}$ & --- & ---- & 1,0548 & 0,735 & 31,679 & 59,11 \\
\hline 19 & $-41,3428^{* *}$ & $32,0970^{* *}$ & --- & --- & --- & 0,635 & 37,171 & 69,36 \\
\hline 20 & $-11,7916^{* *}$ & $16,1602^{* *}$ & --- & --- & --- & 0,625 & 37,652 & 70,26 \\
\hline 21 & $18,4521^{* *}$ & $32,2910^{* *}$ & --- & ---- & --- & 0,611 & 38,376 & 71,60 \\
\hline 22 & $18,4521^{* *}$ & $16,1455^{* *}$ & ---- & ---- & ---- & 0,611 & 38,376 & 71,60 \\
\hline
\end{tabular}

Nivel de significancia: $*=P<0,05 ; * *=P<0,01 ; \mathrm{ns}=$ no significativo.

Donde: $b_{i}=$ coeficientes de las variables independientes para las ecuaciones ajustadas; FCM $=$ factor de corrección de Mayer; $R^{2} a j=$ coeficiente de determinación ajustado; Syx = Error estándar de la estimación.

Cuadro 5. Coeficientes de las ecuaciones obtenidas a través del proceso de selección "forward stepwise" y sus respectivas estadísticas de ajuste y precisión.

Coefficients of the equations obtained through the forward stepwise process and their respective adjustment and precision statistics.

\begin{tabular}{ccccccccccc}
\hline Ecuación & b0 & b1 & b2 & b3 & b4 & b5 & FCM & R$^{2}$ aj & Syx & Syx\% \\
\hline 34 & $4,6291^{* *}$ & $-0,0971^{* *}$ & $0,1100^{* *}$ & $1,2300^{* *}$ & $-0,0171^{* *}$ & $0,0739^{* *}$ & ---- & 0,994 & 4,814 & 8,98 \\
35 & $4,8575^{* *}$ & $-0,0231^{* *}$ & $0,6315^{* *}$ & $-0,2677^{* *}$ & $-4,1018^{* *}$ & & 1,001 & 0,992 & 5,448 & 10,17 \\
\hline
\end{tabular}

Nivel de significancia: $*=P<0,05 ; * *=P<0,01 ; \mathrm{ns}=$ no significativo.

Donde: $b_{i}=$ coeficientes de las variables independientes para las ecuaciones ajustadas; FCM $=$ factor de corrección de Mayer; $R^{2} a j=$ coeficiente de determinación ajustado; $\operatorname{Syx}=$ Error estándar de la estimación. 
el modelo utilizado para estimar el volumen del rodal de las especies secundarias en bracatingais es preciso. Al calcular nuevamente el error estándar (Syx\%) para los datos de validación, resulta en un valor de 8,65 \%. En la figura 2 se encuentran los valores de dispersión residual y el volumen total estimado de leña por hectárea para los datos utilizados en la validación del mejor modelo, donde puede ser observado la distribución homogénea en toda la línea de regresión.

\section{DISCUSIÓN}

Correlación entre las variables utilizadas en el ajuste de los modelos. Diferente del observado por Machado et al. (2008) y por Urbano et al. (2017) para bracatinga, los volúmenes de madera por hectárea de las especies secundarias en bracatingais tienen una buena correlación con la edad del rodal de bracatinga; este hecho se explica por lo que fue observado por Silva et al. (2016), donde a lo largo de los años la bracatinga va siendo sustituida por especies secundarias; es decir, como el número de árboles de especies secundarias aumenta con el paso de los años, el volumen acompaña a esta tendencia, generando una correlación positiva de 0,729 con el volumen en escala aritmética, y de 0,794 con el volumen en escala logarítmica, como se muestra en el cuadro 2. Para las variables $\mathrm{N}, \mathrm{y} d g$, que son variables medidas en campo o calculadas, también se observó una buena correlación con el volumen de las especies secundarias, diferente de lo observado para los volúmenes de bracatinga.
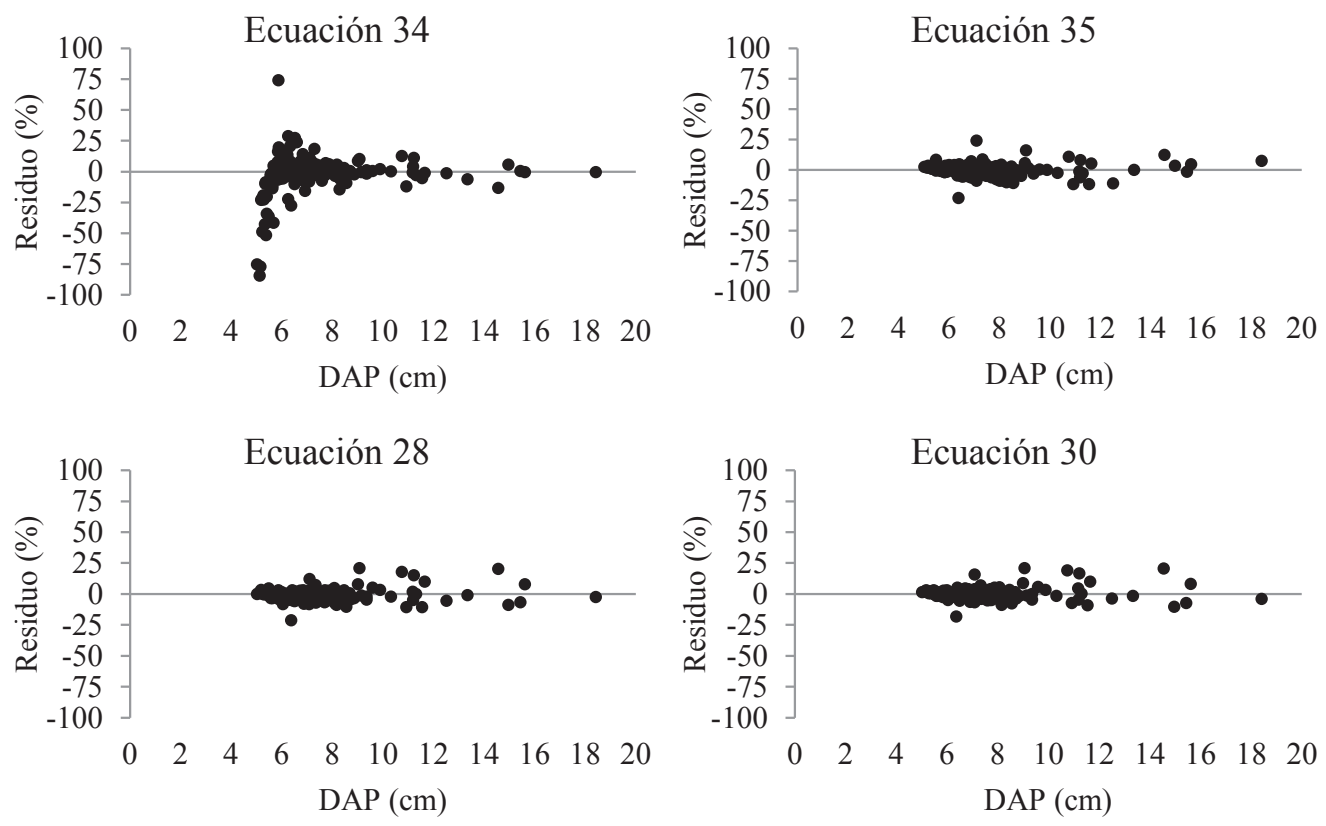

Figura 1. Gráfico de residuos para las cuatro mejores ecuaciones ajustadas para estimación del volumen por hectárea para las especies secundarias en bracatingais.

Graphics of residuals for the four best equations fitted to estimate the volume per hectare for the secondary species in bracatinga forests.
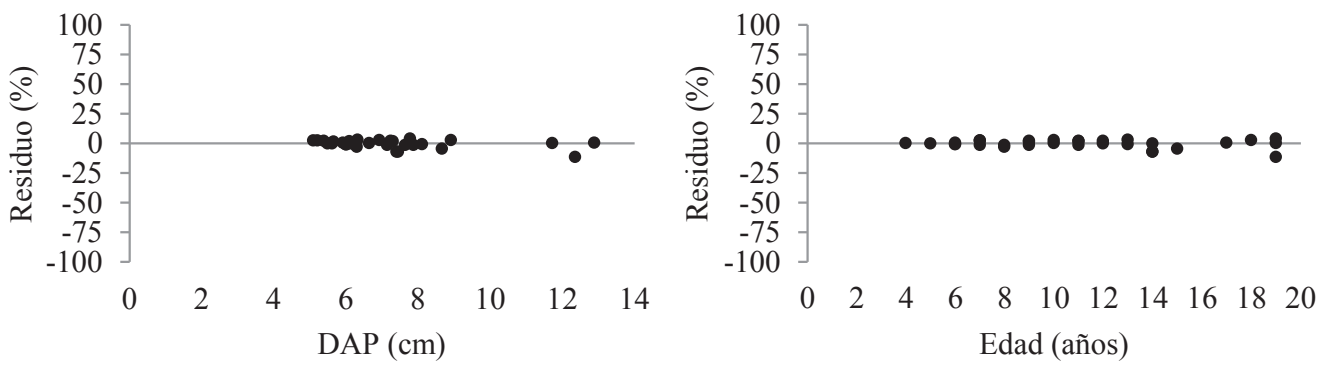

Figura 2. Gráfico de residuos de los datos utilizados para validar la ecuación seleccionada para estimar el volumen por hectárea para las especies secundarias.

Graphics of residuals of the data used to validate the best fitted equation to estimate volume per hectare for the secondary species in bracatinga forests. 
En contraste, la altura media y altura dominante, similar a lo observado para la bracatinga, no presentaron una buena correlación con las variables volumétricas analizadas.

Oliveira et al. (2005), trabajando con fragmentos de bosque en Viçosa (Estado de Minas Gerais, Brasil), señalaron que el área basal mostró una correlación de 0,90 con el volumen total por hectárea y de 0,88 con el volumen de fuste (comercial) por hectárea. Estos autores también observaron una buena correlación del volumen con la altura total y la altura del fuste de los árboles.

Souza et al. (2013), trabajando con modelación de carbono orgánico por hectárea para la bracatinga y Souza et al. (2014), trabajando con modelación de la biomasa aérea por hectárea de la bracatinga, observaron en ambos casos que las variables más correlacionadas fueron las que poseen el área basal combinada con el diámetro medio y con el diámetro cuadrático. Este resultado difiere de los tradicionalmente encontrados en la literatura y con lo observado en el cuadro 2 para las variables volumétricas.

Ajuste de los modelos. Como se observa en el cuadro 4, la mejor ecuación entre los 29 modelos escogidos de la literatura para estimar el volumen de leña por hectárea de las especies secundarias fue el modelo 28 no lineal de Ung y Ouellett (1991). En el trabajo realizado por Machado et al. (2008), en el cual ajustaron ecuaciones para estimar el volumen de leña por hectárea solamente para la bracatinga, observaron que la mejor ecuación fue la ecuación del modelo 11 de Spurr (1952) y en el trabajo realizado por Urbano et al. (2017) en el cual modelaron de nuevo el volumen de leña por hectárea para la bracatinga, observaron que la mejor ecuación ajustada fue la ecuación del modelo 16 de Takata (1959). En la presente investigación la ecuación del modelo 11 quedó en el puesto $12^{\circ}$ en el desempeño general, con error estándar $14,1 \%$ más que el primer lugar y el modelo 16 quedó en el puesto $7^{\circ}$, con error estándar 4,7\% más que el primer lugar.

En trabajo de modelación de volumen del fuste para rodales en bosques nativos en el municipio de Viçosa, Minas Gerais, Brasil, realizado por Oliveira et al. (2005), encontraron que la mejor ecuación corresponde al modelo logaritmizado 29 de Ung y Ouellett (1991). Para estimar el volumen total, en ese mismo estudio observaran que el mejor modelo fue el 28 no lineal de Ung y Ouellett (1991). Entre tanto, en la presente investigación la ecuación del modelo 29 quedó en el puesto $16^{\circ}$ en el desempeño general, con error estándar 19,8\% más que el primer lugar.

Souza et al. (2014), en la modelación de la biomasa de bracatinga en rodales nativos en la región de Curitiba y, Cysneiros et al. (2016) en la modelación del volumen en el bosque húmedo tropical del Amazonas, seleccionaron el modelo de la variable combinada de Spurr (modelo 11) como el más eficiente para la estimación de la producción de leña por unidad de área, y la producción del volumen de madera extraíble, respectivamente. Sin embargo, en el cuadro 4 puede observarse que la ecuación del modelo 11 alcanzó el puesto $12^{\circ}$ en el rendimiento general, con error estándar $14,1 \%$ más que el primer lugar.

Al comparar los 29 modelos tomados de la literatura con las ecuaciones ajustada a través del proceso de selección "forward stepwise", se observa que esta metodología resultó en ecuaciones con los niveles de ajuste y precisión más altos. En la presente investigación la ecuación correspondiente al modelo 28 no lineal de Ung y Ouellett (1991), que mostró el mejor resultado entre los 29 modelos tomados de la literatura, presentó un error estándar 2,77\% mayor que la ecuación logarítmica ajustada a través del proceso de selección de variables "forward stepwise".

Este comportamiento para las ecuaciones obtenidas por proceso de selección de variables está dentro de lo esperado para el uso de esa metodología y conforme a lo observado en los trabajos de Machado et al. (2008), Souza et al. (2014), Cysneiros et al. (2016) y Urbano et al. (2017). En dicho proceso de selección, el algoritmo selecciona variables independientes que presentan alto poder explicativo de la variable dependiente, las cuales no son alto correlacionadas.

Otro aspecto a considerar, es que las variables seleccionadas tienen la capacidad de explicar el comportamiento, casi potencial, de cómo los datos volumétricos están distribuidos a lo largo de las edades en las parcelas muestreadas. Estos factores, explican el hecho del modelo seleccionado presentar bajo error estándar y alta precisión en la estimación del volumen de leña por hectárea de las especies secundarias de los bracatingais.

\section{CONCLUSIONES}

De los 29 modelos tomados de la literatura, la ecuación correspondiente al modelo 28 no lineal de Ung y Ouellett muestra el mejor resultado. Sin embargo, entre todas, la mejor ecuación ajustada es la logarítmica obtenida a través del proceso de selección "forward stepwise".

La prueba de validación de la ecuación logarítmica obtenida por el proceso "forward stepwise" no muestra ninguna diferencia estadística entre los valores reales y estimados, por lo tanto, su uso es adecuado para la estimación, con precisión y confiabilidad, del volumen de leña por hectárea en el procesamiento de los inventarios de las especies secundarias presentes en los bracatingais de la región metropolitana de Curitiba.

Con base en los resultados observados en esta investigación y en resultados encontrados en la literatura, se verifica que la estimación del volumen de rodales a través de modelos matemáticos por unidad de área es una forma precisa y más rápida que el método tradicional de estimar el volumen individual por árbol, siendo ventajoso para la reducción del trabajo de campo y el procesamiento de los datos y por lo tanto resulta en inventarios forestales con menor costo.

Como recomendación futura, alguna mejora en este trabajo podría lograrse con el uso de ecuaciones de volumen individual obtenidas a partir de árboles cubicados en estos rodales, donde se considere la diversidad de especies secundarias que surgen a lo largo de los años en los bracatingais. 


\section{REFERENCIAS}

Burkhart HE, M Tomé. 2012. Modeling forest trees and stands. New York, USA. Springer Science. 457 p.

Caviglione JH, LR Kiihl, PH Caramori, D Oliveira. 2000. Cartas Climáticas do Estado do Paraná. Instituto Agronômico do Paraná - IAPAR, Londrina, 2000. Consultado 30 mar. 2016. Disponible en http://www.iapar.br/modules/conteudo/conteudo.php? conteudo $=677$

Cysneiros VC, SA Machado, AL Pelissari, A Figueiredo Filho, E Urbano. 2016. Modeling of the commercial volume stock in an Ombrophilous forest in the Southwest of the Amazon. Revista Cerne 22(4): 457-464. DOI: 10.1590/01047760201622032204.

Figueiredo Filho A, SA Machado, ROV Miranda, FAS Retslaff (eds.). 2014. Compêndio de Equações de volume e de afilamento de espécies florestais plantadas e nativas para as regiões geográficas do Brasil. Curitiba, Brasil. Universidade Estadual do Centro-Oeste. 306 p.

Figueiredo Filho A, SA Machado, NC Rosot. 1982. Estimativas volumétricas por unidade de área em florestas naturais. Silvicultura em São Paulo 16: 491-504.

Machado SA. 1973. Aplicabilidade de equações de regressão em inventários florestais. In $2^{\circ}$ Congresso Florestal Brasileiro, Curitiba, Brasil. FIEP. p. 175-177.

Machado SA, SJ Téo, E Urbano, MA Figura, LCR Silva. 2006. Comparação de métodos de cubagem absolutos com o volume obtido pelo xilômetro para bracatinga (Mimosa scabrella Bentham). Revista Cerne 12(3): 239-253.

Machado SA, LP Aguiar, A Figueiredo Filho, HS Koehler. 2008. Modelagem do volume do povoamento para Mimosa scabrella Benth. na Região Metropolitana de Curitiba. Revista Árvore 32(3): 465-478.

Machado SA, RF Souza, E Jaskiu, R Cavalheiro. 2011. Construction of site curves for native Mimosa Scabrella stands in the Metropolitan Region of Curitiba. Revista Cerne 17(4): 489-497.

Machado SA, W Pizatto. 1998. Tabela de volume do povoamento para florestas nativas de Araucária angustifolia nos estados da região sul do Brasil. Revista Floresta 26(1): 11-26.

Mazza CADS, AJ Baggio, AA Carpanezzi. 2000. Distribuição espacial da bracatinga na região metropolitana de Curitiba com imagens de satélite Landsat. Colombo,Brasil. Embrapa Florestas. 22 p.

Oliveira ML, CPB Soares, AL Souza, HG Leite. 2005. Equações de volume do povoamento para fragmentos florestais naturais do município de Viçosa, Minas Gerais. Revista Árvore 29(2): 213-225.

Prodan M, R Peters, F Cox, P Real. 1997. Mensura forestal. San José, Costa Rica. Instituto Interamericano de Cooperación para la Agricultura - IICA. 586 p.

Ribeiro A, SA Machado, S Péllico Netto, A Figueiredo Filho. 2012. Quantificação e modelagem dos macronutrientes em povoamentos de bracatinga. Pesquisa Florestal Brasileira 32(69): 29-43. DOI: 10.4336/2012.pfb.32.69.29.

Rosot MAD, SA Machado, NC Rosot. 1990. Estudo de modelos matemáticos para estimar o volume por hectare em um povoamento de Pinus taeda L. em Piraí do Sul - PR. In $6^{\circ}$
Congresso Florestal Brasileiro, Campos do Jordão. Anais do $6^{\circ}$. Congresso Florestal Brasileiro 3: 126-138.

Santos K, CR Sanquetta, RL Eisfield, LF Watzlawick, MAB Ziliotto. 2006. Equações volumétricas por classe diamétrica para algumas espécies folhosas da Floresta Ombrófila Mista no Paraná, Brasil. Revista de Ciências Exatas e Naturais 8(1): 99-112.

Scolforo JRS. 1997. Biometria florestal 2: técnicas de regressão aplicada para estimar: volume, biomassa, relação hipsométrica e múltiplos produtos da madeira. Lavras, Brasil. Universidade Federal de Lavras UFLA/FAEPE. 292 p.

Scolforo JRS. 2005.Biometria Florestal: Parte I - Modelos de regressão linear e não-lienar. Parte II - Modelos para relação hipsométrica, volume, afilamento e peso da matéria seca. Lavras, Brasil. Centro de Editoração FAEPE. 352 p.

Silva JA. 1979. Estimativa do volume por hectare e sua composição em povoamentos de Eucalyptus spp nos cerrados em Minas Gerais e Mato Grosso do Sul. Curitiba. Curitiba, Brasil. Mestrado em Engenharia Florestal - Setor de Ciências Agrárias, Universidade Federal do Paraná. 85 p.

Silva JA. 1985. Modelo de equação para estimar o volume por hectare de Eucalyptus spp na região de Campo Grande, no estado do Mato Grosso do Sul. Floresta e Ambiente 2: 51-59.

Silva LCR. 2013. Estrutura e florística de bracatingais da região metropolitana de Curitiba, Paraná: um enfoque sucessional. Tesis Magíster en Ingeniería Forestal. Curitiba, Brasil. Universidade Federal do Paraná. 125 p. Consultado 01 nov. 2017. Disponible em http://www.acervodigital.ufpr.br/Diss_Silva_2013.

Silva LC, SA Machado, F Galvão, A Figueiredo Filho. 2016. Evolução estrutural de bracatingais da região metropolitana de Curitiba, Paraná, Brasil. Ciência Florestal 261: 171-179. DOI: 10.5902/1980509821102.

Soares CPB, JCC Campos. 2000. Conversão do volume total de povoamentos de eucalipto em volumes comerciais. Revista Árvore 24(4): 477-481.

Souza RF,SA Machado, A Figueiredo Filho. 2013. Modelagem do carbono orgânico total e da lenha por unidade de área para bracatingais nativos. Ciência Florestal 23(1): 117-127.

Souza RF,SA Machado, A Figueiredo Filho.2014. Modelagem da biomassa total e da lenha por unidade de área para bracatingais nativos. Revista Árvore 38(1): 53-62.

Spurr SH. 1952. Forest Inventory. New York, USA. The Ronald Press Company. 476 p.

Stoate TN. 1945. The use of a volume equation in pine stands. Australian Forestry 9: 48-52.

Takata K. 1959. Studies of the volume estimate by basal area at breast height. Journal of the Japanese Forestry Society 41(7): 257-261.

Tukey JW. 1977. Exploratory data analysis. Reading, Massachusetts, USA. Addison-Wesley.

Ung CH, D Ouellet. 1991. Stand volume tables: application to black spruce stands of Lebel-sur-QuÈvillon. Forestry Chronicle 67(6): 712-715.

Urbano E, SA Machado, A Figueiredo Filho, CR Sanquetta. 2017. Stand volume modeling of Mimosa Scabrella in native bracatinga forests. Australian Journal of Basic and Applied Sciences 11(10): 67-77. 
